\title{
Exploring genome-wide - dietary heme iron intake interactions and the risk of type 2 diabetes
}

\section{Louis R. Pasquale ${ }^{1,2}$, Stephanie J. Loomis ${ }^{1,2}{ }^{*}$, Hugues Aschard ${ }^{3}$, Jae H. Kang ${ }^{1}$, Marilyn C. Cornelis ${ }^{1,4}$, Lu Qi $^{1,4}$, Peter Kraft ${ }^{1,3}$ and Frank B. Hu ${ }^{1,3,4}$}

1 Department of Medicine, Channing Division of Network Medicine, Brigham and Women's Hospital, Harvard Medical School, Boston, MA, USA

${ }^{2}$ Glaucoma Service, Massachusetts Eye and Ear Infirmary, Boston, MA, USA

${ }^{3}$ Department of Epidemiology, Harvard School of Public Health, Boston, MA, USA

${ }^{4}$ Department of Nutrition, Harvard School of Public Health, Boston, MA, USA

\section{Edited by:}

Karen T. Cuenco, University of

Pittsburgh, USA

\section{Reviewed by:}

Alexis C. Frazier-Wood, University of Alabama at Birmingham, USA

Cheryl L. Thompson, Case Western

Reserve University, USA

*Correspondence:

Stephanie J. Loomis, Glaucoma Service, Massachusetts Eye and Ear Infirmary, 243 Charles Street, Boston, MA 02114, USA.

e-mail: nhst।@channing.harvard.edu
Aims/hypothesis: Genome-wide association studies have identified over 50 new genetic loci for type 2 diabetes (T2D). Several studies conclude that higher dietary heme iron intake increases the risk of T2D. Therefore we assessed whether the relation between genetic loci and T2D is modified by dietary heme iron intake.

Methods: We used Affymetrix Genome-Wide Human 6.0 array data [681,770 single nucleotide polymorphisms (SNPs)] and dietary information collected in the Health Professionals Follow-up Study ( $n=725$ cases; $n=1,273$ controls) and the Nurses' Health Study ( $n=1,081$ cases; $n=1,692$ controls). We assessed whether genome-wide SNPs or iron metabolism SNPs interacted with dietary heme iron intake in relation to T2D, testing for associations in each cohort separately and then meta-analyzing to pool the results. Finally, we created 1,000 synthetic pathways matched to an iron metabolism pathway on number of genes, and number of SNPs in each gene. We compared the iron metabolic pathway SNPs with these synthetic SNP assemblies in their relation to T2D to assess if the pathway as a whole interacts with dietary heme iron intake.

Results: Using a genomic approach, we found no significant gene-environment interactions with dietary heme iron intake in relation to T2D at a Bonferroni corrected genome-wide significance level of $7.33 \times 10^{-8}$ (top SNP in pooled analysis: intergenic rs 10980508; $\left.p=1.03 \times 10^{-6}\right)$. Furthermore, no SNP in the iron metabolic pathway significantly interacted with dietary heme iron intake at a Bonferroni corrected significance level of $2.10 \times 10^{-4}$ (top SNP in pooled analysis: rs1805313; $p=1.14 \times 10^{-3}$ ). Finally, neither the main genetic effects (pooled empirical $p$ by SNP $=0.41$ ), nor gene dietary heme-iron interactions (pooled empirical $p$-value for the interactions $=0.72$ ) were significant for the iron metabolic pathway as a whole.

Conclusions: We found no significant interactions between dietary heme iron intake and common SNPs in relation to T2D.

Keywords: type 2 diabetes, gene environment interactions, dietary heme iron, pathway analysis

\section{INTRODUCTION}

Type 2 diabetes (T2D) is a multifactorial condition whereby insulin resistance and beta-cell dysfunction produce glucose metabolism alterations, most notably hyperglycemia, resulting in microvascular and macrovascular complications. T2D affects over 25 million individuals (greater than $8 \%$ of the U.S. adult population; American Diabetes Association, 2011) Discovery of the combination of genetic and environmental factors contributing to T2D is essential so that more targeted preventive and management strategies can be devised.

Abbreviations: 1-df test, one degree of freedom gene-environment interaction test; 2-df test, two degree of freedom gene-environment interaction test; HH, hereditary hemochromatosis; HPFS, Health Professionals Follow-Up Study; NHS, Nurses' Health Study; QC, quality control; SNP, single nucleotide polymorphism; T2D, type 2 diabetes.
Dietary heme iron intake is the iron derived from hemoglobin, the protein in the red blood cells found in animal foods such as red meat, fish, and poultry. A recent meta-analysis demonstrated that non-heme iron intake (such as iron derived from vegetables), total iron (iron from heme and non-heme sources) or iron supplements were not associated with increased risk of T2D (Bao et al., 2012). However, epidemiologic studies indicate that increased total body iron stores are associated with an increased risk of T2D (Salonen et al., 1998; Jiang et al., 2004b). While the relation between dietary heme iron intake and obesity has not been well-studied, diets with higher heme iron intake have consistently been associated with increased risk of T2D (Jiang et al., 2004a; Lee et al., 2004; Song et al., 2004; Rajpathak et al., 2006). Iron is a catalyst in the formation of hydroxyl radicals, which are powerful pro-oxidants that attack cellular membrane lipids, proteins, and nucleic acids (Nelson, 1999). While heme iron intake is not directly linked to 
obesity, excess iron stores produced by dietary behaviors may render pancreatic beta cells particularly vulnerable to oxidative stress because they possess a weak antioxidative stress system (Tiedge et al., 1997).

While dietary heme iron intake plays a role in T2D, genomewide association studies have revealed approximately 50 genetic loci for T2D (Visscher et al., 2012), raising the question of whether gene environment interactions focused on dietary heme iron intake exist in T2D.

In fact, a previous candidate gene association study demonstrated a significant interaction between dietary iron intake and the $\mathrm{H} 63 \mathrm{D}$ or $\mathrm{C} 282 \mathrm{Y}$ risk variants for HFE, the gene for hereditary hemochromatosis $(\mathrm{HH})$, in relation to T2D risk (Qi et al., 2005). HH is a condition where secondary diabetes mellitus is a well-known complication that results from high iron stores in the pancreas (Utzschneider and Kowdley, 2010). In this work, we assessed whether the relation between dietary heme iron intake and T2D is modified by genome-wide single nucleotide polymorphisms (SNPs) or iron metabolic pathway SNPs.

\section{MATERIALS AND METHODS STUDY POPULATION}

This study included participants from two longitudinal cohort studies, the Nurses' Health Study (NHS) and the Health Professionals Follow-up Study (HPFS; Barton et al., 1980; Rimm et al., 1990). The HPFS and the NHS are two populations of men and women, respectively, for whom stored blood, DNA samples, and dietary heme iron intake data are available. The NHS began in 1976 with 121,700 female registered nurses aged 30-55, and the HPFS started in 1986 with 51,529 male health professionals aged 40-75. Both cohorts have been followed-up biennially through mailed questionnaires that gather data on new diseases, diet, and other lifestyle factors.

\section{ASCERTAINMENT OF TYPE 2 DIABETES}

For participants with a self-report of diabetes on biennial questionnaires, we mailed supplemental questionnaires inquiring about the diagnosis and treatment of their condition, as well as a history of ketoacidosis to corroborate the self-report and to differentiate between type 1 diabetes mellitus and T2D. We applied criteria established by the National Diabetes Data Group until 1997(National Diabetes Data Group, 1979), and then used the revised criteria of the American Diabetes Association from 1998 onward to these supplemental questionnaires (Report of the expert committee on the diagnosis classification of diabetes mellitus, 1997). This approach was found to be valid for identifying T2D as medical record review confirmed $98 \%$ of cases in a subsample (Manson et al., 1991).

\section{FORMATION OF DOMESTIC CASE-CONTROL GROUPS AND GENOTYPING}

We accrued 2,591 cases with T2D and 3,508 controls with completed high throughput genotyping from 1986 to 2006 in HPFS and 1980 to 2006 in NHS. From these cases we chose participants of European descent with dietary questionnaire responses prior to diagnosis of T2D. Controls were not strictly matched to cases because some subjects did not consent to have their genotyping data posted on dbGap. Thus we chose controls with complete dietary data that were age matched to cases. Ultimately, 1,806 cases and 2,965 controls were eligible for the study (see Table 1). Informed consent was obtained from all participants in this study and the institutional review board at the Harvard School of Public Health approved the study.

We completed genotyping on the Affymetrix Genome-Wide Human 6.0 array at the Broad Institute (Cambridge, MA, USA). Details regarding the quality control measures employed in genotyping these samples have been previously published (Qi et al., 2010). After data cleaning, 706,034 SNPs remained for analysis on 725 cases and 1,273 controls in HPFS. In NHS, a total of 704,409 SNPs remained after data cleaning and were analyzed on 1,081 cases and 1,692 controls.

\section{DATA ANALYSIS}

To assess the interaction between dietary heme iron intake and gene variants in relation to $\mathrm{T} 2 \mathrm{D}$, we created the following logistic regression models:

(1) $\mathrm{T} 2 \mathrm{D}=\beta_{0}+\beta_{1}$ (age) $+\beta_{2}$ (body mass index, BMI) $+\beta_{3}$ (heme) - Environmental model

(2) $\mathrm{T} 2 \mathrm{D}=\beta_{0}+\beta_{1}(\mathrm{SNP})+\beta_{2}$ (age) $+\beta_{3}(\mathrm{BMI})+\beta_{4}$ (heme) Genetic model

(3) $\mathrm{T} 2 \mathrm{D}=\beta_{0}+\beta_{1}(\mathrm{SNP})+\beta_{2}($ age $)+\beta_{3}(\mathrm{BMI})+\beta_{4}$ (heme) $+\beta_{5}$ $(\mathrm{SNP} \times$ heme $)$ - one-degree of freedom gene-environment (GxE) interaction model

Except for Model 1 (which we generated in SAS version 9.3, Cary, NC, USA), we formulated these models in each cohort separately using PLINK. Heme iron intake (mg/day), BMI $\left(\mathrm{kg} / \mathrm{m}^{2}\right.$, as of 1986 for HPFS and 1980 for NHS) and age (years, as of 1986 for HPFS and 1980 for NHS) were treated as continuous variables. We transformed heme to be centered on the mean value for each cohort. SNPs were coded as 0,1 , or 2 minor alleles. We controlled for the top three eigenvectors in NHS and the top four eigenvectors in HPFS. We also tested models that treated the dietary heme iron intake term as a dichotomous variable divided at the median value in controls.

To test for interactions between dietary heme intake and SNP genotypes in relation to T2D, we utilized a one degree of freedom

Table 1 | Characteristics of Health Professionals Follow-up Study (HPFS) and Nurses' Health Study (NHS) cohorts.

\begin{tabular}{llllll}
\hline Cohort & $\boldsymbol{N}$ & Mean heme, SD (mg/day) & Mean body mass index, SD (kg/m $\left.{ }^{2}\right)$ & Mean age at baseline, SD (years) \\
\hline HPFSCasesControls & 19987251273 & $1.29(0.43) 1.39(0.43) 1.23(0.42)$ & $25.05(3.46) 27.87(3.82) 25.01(2.74)$ & $54.19(8.36) 52.93(8.16) 54.91(8.39)$ \\
NHSCasesControls & 277310811692 & $1.36(0.45) 1.41(0.44) 1.33(0.45)$ & $26.08(5.00) 28.66(4.95) 24.43(4.29)$ & $47.47(6.76) 47.31(6.73) 47.57(6.78)$
\end{tabular}

$S D$, standard deviation. 
(1-df) test, which is represented as $\beta_{5}$ of Model 3. Models 1 and 2 tested the marginal effects of dietary heme iron intake and SNPs respectively, adjusting for age and BMI. We also performed a two degree of freedom joint test (2-df) by comparing the fit of the null model containing the environment exposure only (Model 1) to the model with gene and gene-environment covariates (Model 3) as an alternate test of the gene-environment interaction (Cornelis et al., 2011; Manning et al., 2011). After running these models separately in NHS and HPFS, we performed tests for heterogeneity of the cohort specific results to check for appropriateness of pooling the data. We conducted an inverse variance-weighted fixed-effects meta-analysis of estimates from the two cohorts using the METAL software. ${ }^{1}$ Only SNPs with genotypes available in both cohorts were included in the meta-analysis $(N=681,770)$.

Next we limited our gene-environment interaction analyses to SNPs in the iron metabolic pathway created using the KEGG database ${ }^{2}$ and other sources (Michal, 1999; Andrews and Schmidt, 2007; Andrews, 2008; Iron Health Alliance, 2009; William, 2009). We identified 237 SNPs in genes coding for enzymes in this pathway that were present on the Affymetrix 6.0 platform (Table 2). We repeated the logistic regression analysis of Models 2 and 3 using the iron metabolic pathway SNPs. We used a Bonferroni correction based on the number of SNPs analyzed to establish statistical significance in genome-wide $\left(p=0.05 / 681,770=7.33 \times 10^{-8}\right)$ and pathway analyses $\left(p=0.05 / 237=2.10 \times 10^{-4}\right)$. These estimates of the correction for multiple comparisons are somewhat liberal in that they do not account for the secondary analyses we performed.

To evaluate whether the iron metabolic pathway as a whole might interact with dietary heme intake, we assessed whether the iron metabolic pathway SNP panel was enriched with variants strongly associated with T2D. We compared the distribution of $p$-values in the SNPs from the iron metabolic pathway with the distribution of SNPs from 1,000 permuted "synthetic pathways" generated by randomly picking SNPs available on the platform that were not in the iron metabolic pathway. To ensure an adequate comparison, all synthetic pathways were constructed such that they had the same number of genes and the same number of SNPs per gene $\pm 10 \%$ as the heme pathway. Enrichment of SNPs with low $p$-values was evaluated by comparing $C_{\mathrm{obs}}$, the count of SNPs with a $p$-value below a given significance threshold $T$ in the heme metabolic pathway, to $C_{\text {syn }}$, the corresponding count derived in the synthetic pathways. The empirical $p$-value (for both the main genetic effect and the gene-environment interaction term) related to enrichment for a given $T$ was derived as the number of times $C_{\text {obs }}$ was higher than $C_{\text {syn }}$ divided by 1,000 , the total number of synthetic pathways.

\section{RESULTS}

Type 2 diabetes cases were similar in age compared to controls in men (overall mean $\pm \mathrm{SD}=54.3 \pm 8.4$ years) and women ( $47.5 \pm 6.8$ years). As expected, cases had higher BMI and higher mean dietary heme iron intake than controls in men and women (Table 1). Dietary heme iron intake was adversely associated

\footnotetext{
${ }^{1}$ www.sph.umich.edu/csg/abecasis/metal/

${ }^{2}$ www.genome.jp/kegg/pathway.html
}

with $\mathrm{T} 2 \mathrm{D}\left[\mathrm{OR}=1.36(1.17,1.58)\right.$; pooled $p=7.51 \times 10^{-5}$; Model 1]. As expected, the top SNP associated with T2D was in TCF7L2 (rs7901695; pooled $p$-value $=1.88 \times \times 10^{-14}$ ) (Model 2).

Using the 1-df test (Model 3), no gene-environment interaction achieved genome-wide significance level. The most significant interaction with continuous dietary heme iron intake was rs10980508 (pooled $p=1.03 \times 10^{-6}$; an intergenic SNP between muscle, skeletal, receptor tyrosine kinase (MUSK) and Sushi, von Willebrand factor type A, EGF, and pentraxin domains-containing 1 (SVEP1; Table 3). The 2-df test revealed that SNPs in TCF7L2 had genome-wide margin association with T2D but did not reveal new marginal gene effects of genome-wide significance; nor was there significant interaction between TCF7L2 and dietary heme iron intake in T2D (data not shown). When we generated models substituting dietary heme intake with red meat, processed meat, and total meat, we found similar results with top marginal genetic effects in TCF7L2; yet, Model 3 did not yield significant gene-environment interactions (data not shown).

No significant iron metabolism SNP - dietary heme iron intake interaction was detected with the 1-df test (Model 3) in relation to T2D (top SNP rs1805313; in ALAD (delta-aminolevulinate dehydratase); pooled $p=1.14 \times 10^{-3}$; Bonferroni corrected significance level $\left.p=2.10 \times 10^{-4}\right)$. The 2 -df test of gene and gene-environment interactions also did not reveal any significant interactions between dietary heme iron intake and SNPs in the iron metabolic pathway SNP in pooled analyses (data not shown).

Compared with synthetic pathways, the iron metabolic pathway was not associated with T2D when we performed the analyses by SNP (pooled empirical $p$ by SNP $=0.41$ ). Similar null results were obtained when interactions with dietary heme iron intake were considered (pooled empirical $p$-value for the interactions $=0.72$ ). Interactions between various forms of dietary meat intake and the iron metabolic pathway were also not significant.

\section{DISCUSSION}

Neither the 1-df test nor the 2-df test revealed any genomewide significant interactions between dietary heme iron intake and genomic SNPs in T2D. Furthermore, the relation between an iron metabolic pathway SNP panel and T2D was not modified by dietary heme iron intake. Finally the iron metabolic pathway was not enriched with SNPs related to T2D.

There could be several possible reasons for the null results reported here. First, despite its large size $(n=4,771)$ our study could be underpowered to find modest interaction terms. In fact, only in a log additive model would we achieve $80 \%$ power to detect a genome-wide environmental interaction effect of 1.8 (assumes minor allele frequency $=0.4$; genetic relative risk $=1.2$, and relative risk of the highest tertile of dietary heme iron intake $=1.3$ ). Nonetheless we had $\sim 80 \%$ power to discover an interaction effect of 1.5 between iron metabolic SNPs and dietary heme iron intake in relation to $\mathrm{T} 2 \mathrm{D}$ using similar assumptions in a dominant inheritance model. Second, power could be compromised due to inherent error in measuring dietary heme iron intake. Third, self-reported iron intake, while collected using a validated food frequency questionnaire, could be prone to recall bias. Finally, the 
Table 2 | Genes and single nucleotide polymorphisms (SNPs) in the heme iron metabolic pathway on the Affymetrix 6.0 array that passed quality control.

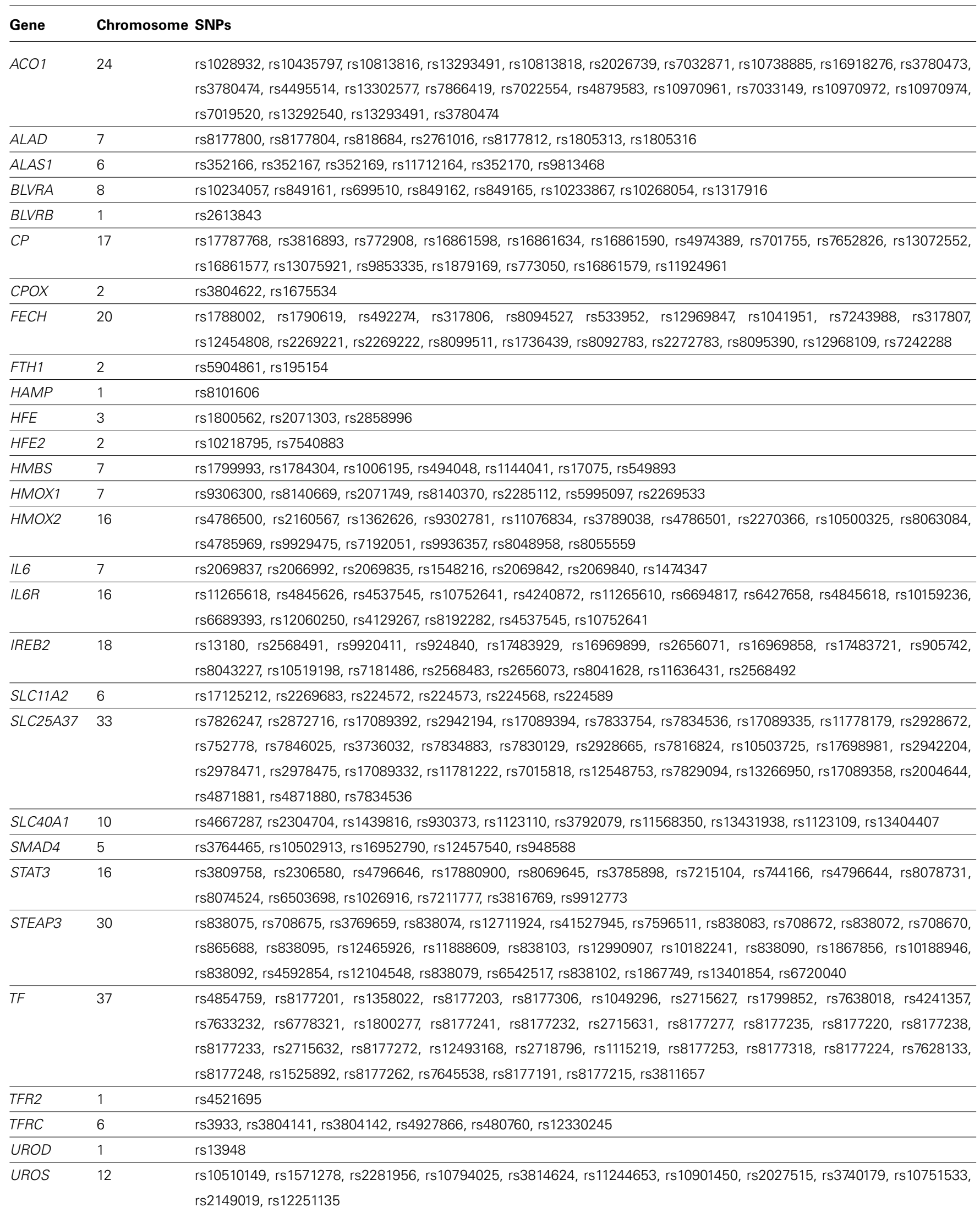


Table 3 | Top 10 -values from genome-wide SNP-heme interactions predicting type 2 diabetes adjusting for age, body mass index, dietary heme iron intake, and eigenvectors using a one degree of freedom test from meta-analysis of the Health Professionals Follow-up Study and Nurses' Health Study. ${ }^{\text {a }}$

\begin{tabular}{|c|c|c|c|c|c|c|c|}
\hline $\begin{array}{l}\text { Chromo- } \\
\text { some }\end{array}$ & SNP & $\begin{array}{l}\text { Beta, } \\
\text { HPFS }^{b}\end{array}$ & $\begin{array}{l}\text { Beta, } \\
\text { NHS }^{b}\end{array}$ & $\begin{array}{l}p \text {-Value, } \\
\text { HPFS }^{b}\end{array}$ & $\begin{array}{l}p \text {-Value, } \\
\text { NHS }^{b}\end{array}$ & $\begin{array}{l}p \text {-Value, } \\
\text { pooled }^{b, c}\end{array}$ & Associated gene \\
\hline 9 & rs10980508 & 0.96 & 0.64 & $1.81 \times 10^{-4}$ & $1.23 \times 10^{-3}$ & $1.03 \times 10^{-6}$ & Upstream of MUSK, downstream of SVEP1 \\
\hline 9 & rs12378245 & 0.96 & 0.61 & $1.77 \times 10^{-4}$ & $1.76 \times 10^{-3}$ & $1.51 \times 10^{-6}$ & Upstream of MUSK, downstream of SVEP1 \\
\hline 9 & rs10817049 & 0.92 & 0.61 & $3.09 \times 10^{-4}$ & $1.61 \times 10^{-3}$ & $2.16 \times 10^{-6}$ & Upstream of MUSK, downstream of SVEP1 \\
\hline 9 & rs7048110 & 0.96 & 0.58 & $1.80 \times 10^{-4}$ & $2.75 \times 10^{-3}$ & $2.53 \times 10^{-6}$ & Upstream of MUSK, downstream of SVEP1 \\
\hline 16 & rs17177078 & 1.48 & 0.83 & $1.50 \times 10^{-4}$ & $5.66 \times 10^{-3}$ & $5.06 \times 10^{-6}$ & Intron in TNRC6A \\
\hline 9 & rs10980495 & 0.91 & 0.57 & $3.60 \times 10^{-4}$ & $3.33 \times 10^{-3}$ & $5.49 \times 10^{-6}$ & Upstream of MUSK, downstream of SVEP1 \\
\hline 7 & rs1525739 & -0.58 & -0.44 & $7.85 \times 10^{-4}$ & $2.11 \times 10^{-3}$ & $6.26 \times 10^{-6}$ & Downstream of $A G R 3$, upstream of $A G R 2$ \\
\hline 9 & rs10448267 & 0.94 & 0.54 & $2.58 \times 10^{-4}$ & $4.95 \times 10^{-3}$ & $6.58 \times 10^{-6}$ & Upstream of MUSK, downstream of SVEP1 \\
\hline
\end{tabular}

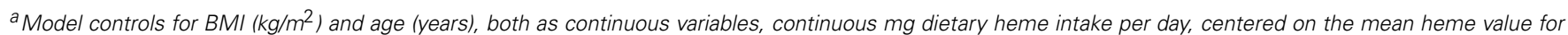
each cohort and eigenvectors 1-3 for NHS or eigenvectors 1-4 for HPFS. SNPs are coded as 0, 1, or 2 minor alleles.

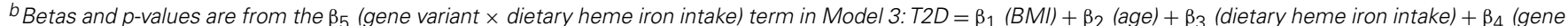
variant $)+\beta_{5}$ (gene variant $\times$ dietary heme iron intake) + eigenvectors

${ }^{c} P$ for heterogeneity is $>0.05$ (lowest $p$-value is 0.4).

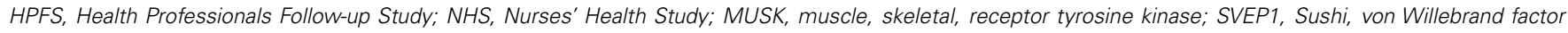

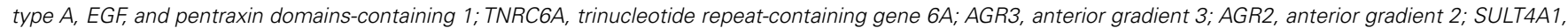
sulfotransferase family $4 A$, member 1 .

reason why a higher dietary heme iron dietary intake increases the risk of T2D could be solely related to environmental influences (Lee et al., 2004).

A prior study, using a candidate approach, did find a marginal interaction between $H F E$ and dietary heme iron intake in T2D $(p=0.03$; Qi et al., 2005). While we did not discover any new gene-dietary heme iron interactions, more studies using serum

\section{REFERENCES}

Andrews, N. C. (2008). Forging a field: the golden age of iron biology. Blood $112,219-230$.

Andrews, N. C., and Schmidt, P. J. (2007). Iron homeostasis. Annu. Rev. Physiol. 69, 69-85.

American Diabetes Association. (2011). Diabetes Statistics. Available at: http://www.diabetes.org (accessed November 26, 2012).

Bao, W., Rong, Y., Rong, S., and Liu, L. (2012). Dietary iron intake, body iron stores, and the risk of type 2 diabetes: a systemic review and metaanalysis. BMC Med. 10:119. doi: 10.1186/1741-7015-10-119

Barton, J., Bain, C., Hennekens, C. H., Rosner, B., Belanger, C., Roth, A., etal. (1980). Characteristics of respondents and nonrespondents to a mailed questionnaire. Am J. Public Health 70, 823-825.

Cornelis, M. C., Tchetgen, E. J., Liang, L., Qi, L., Chatterjee, N., Hu, F. B., et al. (2011). Gene-environment interactions in genome-wide association studies: a comparative study of tests applied to empirical studies of type 2 diabetes. Am. J. Epidemiol. 175, 191-202.

Iron Health Alliance. (2009). Iron Metabolism. Excess Iron. Available at: http://www.excessiron.com/ironmetabolism.jsp (accessed September 23, 2009).

Jiang, R., Ma, J., Ascherio, A., Stampfer, M. J., Willett, W. C., and Hu, F. B. (2004a). Dietary iron intake and blood donations in relation to risk of type 2 diabetes in men: a prospective cohort study. Am. J. Clin. Nutr. 79 $70-75$.

Jiang, R., Manson, J. E., Meigs, J. B., Ma, J., Rifai, N., and $\mathrm{Hu}$, F. B. (2004b). Body iron stores in relation to risk of type 2 diabetes in apparently healthy women. JAMA 291, 711-717

Lee, D. H., Folsom, A. R., and Jacobs, D. R. Jr. (2004).Dietary iron intake and Type 2 diabetes incidence in postmenopausal women: the Iowa

biomarkers as surrogates of dietary heme iron intake might point to new gene-iron intake interactions in T2D.

\section{ACKNOWLEDGMENTS}

This work was supported by grants CA87969, CA49449, CA055075, EY09611, EY015473, DK58845, UO1 HG004728 and U01HG004728-02S1 from the National Institutes of Health.

Women's Health Study. Diabetologia 47, 185-194.

National Diabetes Data Group. (1979). Classification and diagnosis of diabetes mellitus and other categories of glucose intolerance. Diabetes 28 , 1039-1057.

Manning, A. K., LaValley, M., Liu, C. T., Rice, K., An, P., Liu, Y., et al. (2011) Meta-analysis of gene-environment interaction: joint estimation of SNP and SNP $\mathrm{x}$ environment regression coefficients. Genet. Epidemiol. 35113518.

Manson, J. E., Rimm, E. B., Stampfer, M. J., Colditz, G. A., Willett, W. C., Krolewski, A.S., et al. (1991). Physical activity and incidence of noninsulin-dependent diabetes mellitus in women. Lancet 338, 774-778.

Michal, G. (1999). Biochemical Pathways: An Atlas of Biochemistry and Molecular Biology. New York: John Wiley.

Nelson, N. C. (1999). Disorders of iron metabolism. N. Engl. J. Med. 341, 1986-1995.
Qi, L., Cornelis, M. C., Kraft, P., Stanya, K. J., Linda Kao, W. H., Pankow, J. S., et al. (2010). Genetic variants at 2q24 are associated with susceptibility to type 2 diabetes. Hum. Mol. Genet. 19, 2706-2715.

Qi, L., Meigs, J., Manson, J. E., Ma, J., Hunter, D., Rifai, N., et al. (2005). HFE genetic variability, body iron stores, and the risk of type 2 diabetes in U.S. women. Diabetes. 54, 3567-3572.

Rajpathak, S., Ma, J., Manson, J., Willett, W. C., and Hu, F. B. (2006). Iron intake and the risk of type 2 diabetes in women: a prospective cohort study. Diabetes Care 29, 1370_ 1376.

American Diabetes Association. (1997). Report of the Expert Committee on the Diagnosis and Classification of Diabetes Mellitus. Diabetes Care 20, 1183-1197.

Rimm, E. B., Stampfer, M. J., Colditz, G. A., Giovannucci, E., and Willett, W. C. (1990). Effectiveness of various mailing strategies among nonrespondents 
in a prospective cohort study. Am. J. Epidemiol. 131, 1068-1071.

Salonen, J. T., Tuomainen, T. P., Nyyssonen, K., Lakka, H. M., and Punnonen, K. (1998). Relation between iron stores and non-insulin dependent diabetes in men: case-control study. BMJ 317, 727.

Song, Y., Manson, J. E., Buring, J. E., and Liu, S. (2004). A prospective study of red meat consumption and type 2 diabetes in middle-aged and elderly women: the women's health study. Diabetes Care 27, 21082115.

Tiedge, M., Lortz, S., Drinkgern, J., and Lenzen, S. (1997). Relation between antioxidant enzyme gene expression and antioxidative defense status of insulin-producing cells. Diabetes 46, 1733-1742.

Utzschneider, K. M., and Kowdley, K. V. (2010).Hereditary hemochromatosis and diabetes mellitus: implications for clinical practice. Nat. Rev. Endocrinol. 6, 26-33.

Visscher, P. M., Brown, M. A., McCarthy, M. I., and Yiang, J. (2012). Five years of GWAS discovery. Am. J. Hum. Genet. 90 7-24.

William, W. (2009). Flow Chart of Intermediates in the Synthesis of Hemoglobin (accessed September 23, 2009).

Conflict of Interest Statement: The authors declare that the research was conducted in the absence of any commercial or financial relationships that could be construed as a potential conflict of interest.

Received: 23 October 2012; accepted: 08 January 2013; published online: 30 January 2013.

Citation: Pasquale LR, Loomis SJ, Aschard H, Kang JH, Cornelis MC, Qi L, Kraft $P$ and $H u$ FB (2013)
Exploring genome-wide - dietary heme iron intake interactions and the risk of type 2 diabetes. Front. Gene. 4:7. doi: 10.3389/fgene.2013.00007

This article was submitted to Frontiers in Applied Genetic Epidemiology, a specialty of Frontiers in Genetics.

Copyright (C) 2013 Pasquale, Loomis, Aschard, Kang, Cornelis, Qi, Kraft and $H u$. This is an open-access article distributed under the terms of the Creative Commons Attribution License, which permits use, distribution and reproduction in other forums, provided the original authors and source are credited and subject to any copyright notices concerning any third-party graphics etc. 